\title{
Influencia de la tensión media aplicada en la vida a fatiga de un material compuesto vidrio-epoxi unidireccional sometido a cargas cíclicas desviadas respecto a la dirección de la carga
}

\section{Influence of the average stress in a unidirectional glass-epoxi composite material subjected to off-axis cyclic loads}

\author{
D. Revuelta ${ }^{(*)}$
}

Recepción/Received: 1-IV-05

Aceptación/Accepted: 29-VII-05

\section{RESUMEN}

En trabajos anteriores del autor desarrolló un modelo teórico de vida a fatiga para materiales compuestos unidireccionales de matriz epoxi reforzados con fibra de vidrio bajo condiciones generales de carga basado en los modos fundamentales de rotura por fatiga y en criterios de rotura local. Se comprobó que la vida de fatiga dependía de la tensión cíclica de cortadura y de la máxima tensión transversal a la dirección de crecimiento de las grietas. Siguiendo el modelo previo, este trabajo analiza la influencia que tiene la tensión media aplicada en la vida a fatiga de estos materiales.

Palabras clave: composite, refuerzo de fibras, vidrio, polímero, fatiga.

\section{SUMMARY}

A fatigue theoretical model for continuous glass-fibre reinforced epoxy composite material under general load conditions was developed in previous works based on the principal fatigue failure modes and on local failure criteria. It was demonstrated that fatigue life depended on the cyclic shear stress and the maximum stress transverse to the crack growth direction. Following the previous model, this work analyzes the influence of the average applied stress on the fatigue life of the material.

Keywords: composite, fibre reinforcement, glass, polimer, fatigue.

\section{INTRODUCCIÓN}

La fatiga es la condición por la cual un material se agrieta o falla como resultado de esfuerzos repetidos cuyo valor nominal es inferior al de la resistencia estática del material. Como esfuerzos cíclicos se incluyen tanto los que producen cargas externas repetidas, como los esfuerzos térmicos que resultan del calentamiento y enfriamiento alternados. Estas circunstancias se encuentran en la práctica totalidad de las aplicaciones tecnológicas: mecánicas, eléctricas, arquitectónicas, químicas y biomédicas; y afectan a una amplia gama de materiales con los que se construyen dichas aplicaciones: metales y aleaciones, cerámicas, polímeros y materiales compuestos.

Se define la vida de fatiga de un componente o material como el número total de ciclos de esfuerzo necesarios para causar el fallo catastrófico, entendiéndose como tal aquél en el que la aplicación deja de desempeñar la función para la que fue diseñada (1). La gravedad de las consecuencias que acarrea el fallo de un material hace que la vida a fatiga se estudie con frecuencia en el laboratorio,

\footnotetext{
(*) Instituto de Ciencias de la Construcción Eduardo Torroja - CSIC, Madrid (España).
} 
para a posteriori poder aplicar la información obtenida en ensayos controlados a diseños y componentes en servicio real. El método más comúnmente empleado para estudiar la vida a fatiga consiste en someter al material a cargas cíclicas de amplitud constante y registrar el número de ciclos hasta la rotura -pérdida total de la capacidad de carga- de la correspondiente probeta de ensayo.

Los datos provenientes de los ensayos de fatiga generalmente se presentan en forma de las llamadas curvas $\mathrm{S}-\mathrm{N}$, en donde los esfuerzos alternantes, $\mathrm{S}$-máxima tensión nominal aplicada - se representan frente al número de ciclos hasta la rotura, $\mathrm{N}_{\mathrm{R}}$. El estudio de estas curvas revela que, a medida que disminuye el nivel de las tensiones aplicadas, aumenta la cantidad de ciclos hasta la rotura. En aleaciones ferrosas y en algunas aleaciones de titanio se observa la existencia de un límite de esfuerzos por debajo del cual no se produce la rotura por fatiga. A este límite se le conoce como límite de fatiga. Por desgracia, la mayoría de materiales no presentan dicho límite, por lo que con frecuencia se define el límite de resistencia de un material a la fatiga como el máximo esfuerzo aplicado que no producirá la rotura del material antes de transcurridos un millón de ciclos de carga. Como los materiales compuestos de matriz polimérica presentan generalmente una resistencia a la fatiga mayor que la de los metales, el límite de un millón de ciclos es muchas veces una restricción demasiado conservadora.

Pero el principal problema que presentan los materiales compuestos es el de su anisotropía. Debido a la conFiguración interna de un sistema de matriz polimérica reforzada con fibra unidireccional, la dirección de aparición y crecimiento de grietas no es perpendicular a la carga, como en los materiales isótropos (2). Este hecho añade un grado de complejidad que hace que, para determinar la vida a fatiga de un material compuesto, se requiera un número muy elevado de ensayos, con el coste que lleva aparejado.

\section{MODELO TEÓRICO}

En trabajos precedentes del autor (3) se llegó a la conclusión de que la vida de fatiga de un material compuesto unidireccional de vidrio-epoxi se podía expresar como:

$$
f\left(N_{R}\right)=\Delta \tau_{L T}+k \sigma_{T}^{\max }=\sigma^{\prime} N_{R}^{m}
$$

en donde $N_{R}$ es el número total de ciclos hasta la rotura, y $\sigma^{\prime}$ y $m$ son constantes del material, que han de ser determinadas mediante experimentos.

El parámetro $k$ es un parámetro de interacción multiaxial, que combina las contribuciones de cortadura cíclica y tensión transversal. En el caso de un laminado vidrio-epo$\mathrm{xi}$, se dedujo experimentalmente que este parámetro tenía un valor de $k=1$. Al primer término de la Ecuación [1] se le denominó función de tensiones de fatiga crítica, ya que esta función es la que rige la vida del material.

Mediante la transformación de las cargas globales aplicadas a escala de la lámina en tensiones locales a escala de la grieta (Figura 1), la función de tensiones de fatiga crítica se podía expresar en función del ratio de esfuerzos, $R$, definido como $\sigma_{\min } / \sigma_{\max }$ y del ángulo que forma la carga respecto a la dirección de la fibra, $\theta$ :

$$
\Delta \tau_{L T}+k \sigma_{T}^{\max }=((1-R) \cos \theta+k \sin \theta) \sin \theta \sigma_{\max }
$$

donde $\sigma_{\max }$ es la máxima tensión nominal aplicada durante un ciclo de fatiga expresada en coordenadas globales. En el trabajo anterior se estudió la naturaleza de la función de tensiones de fatiga, el efecto de la orientación de la fibra y el efecto del parámetro de interacción de las tensiones multiaxiales para un caso de fatiga biaxial con $R=0$, que se resume en la interpretación de la Figura 2 . Se concluía que existían tres regiones distintas, según los diferentes modos de rotura: una región con predominio de rotura en la fibra, para fibra orientada prácticamente en la dirección de la carga, una región con predominio de rotura por cortadura (fallo en la matriz) y una región con predominio de rotura por tensión transversal (fallo en la matriz).

Otros parámetros críticos a la hora de evaluar la resistencia a fatiga cíclica de un material compuesto son el signo y la magnitud de la tensión media aplicada durante los ciclos de carga. Para un material compuesto unidireccional con la fibra orientada un cierto ángulo $\theta$ respecto al eje de aplicación de la carga, la función de tensión crítica de fatiga se puede expresar en función de la amplitud, $\Delta s$, y del ratio de esfuerzos, $R$, como:

$$
\Delta \tau_{L T}+k \sigma_{T}^{\max }=\left(\cos \theta+\frac{k}{1-R} \sin \theta\right) \sin \theta \Delta \sigma
$$

Según la Ecuación [3], la contribución de la tensión media a la vida de fatiga está gobernada por el ratio $k /(1-R)$. La Figura 3 muestra para un valor de $k=1$ la influencia del ratio de esfuerzos $R$ en un material compuesto vidrio-epoxi. De nuevo se observan tres regiones según el modo de fallo predominante. El límite entre la zona dominada por $\Delta \tau$ y la dominada por $\sigma_{T}$ cambia al variar la tensión media. A medida que el ratio $R$ disminuye, la resistencia a la fatiga de un material compuesto unidireccional aumenta. De la Figura 3 también se desprende que, para ángulos elevados entre la fibra y la dirección de la carga, la función de tensión crítica de fatiga adquiere un carácter más estable, con lo que es de esperar que a partir de los $70^{\circ}$, el comportamiento a fatiga sea similar. Asimismo, se comprueba que, 
para ángulos entre la fibra y la carga pequeños, el modo de rotura se debe a las tensiones de cortadura; a medida que el ratio de esfuerzos crece, aumenta la zona dominada por rotura debida a la tensión transversal.

\section{RESULTADOS Y DISCUSIÓN}

Fawaz y Hellín (4) investigaron la influencia del ratio de esfuerzos $R$ en el comportamiento a fatiga de un sistema vidrio-epoxi unidireccional. La Figura 4 muestra los resultados experimentales para $R=0$ y $R=0,5$, y para diferentes ángulos entre la fibra y la carga. Como era de esperar por lo deducido del estudio de la Figura 3, la vida a fatiga del material compuesto unidireccional disminuye al incrementar la tensión media, es decir, al aumentar el ratio de esfuerzos $R$. Los experimentos realizados para $R=0,5$ revelaron vidas más cortas que los obtenidos con $R=0$. También se desprende que a medida que el ángulo entre la fibra y la dirección de aplicación de la carga aumenta, el material presenta un comportamiento más estable, lo cual es consistente con lo que predice la Figura 3.

Una vez aplicada la ec. [4] a los datos de Fawaz y Ellyin, el mejor ajuste del modelo se obtiene para un valor de $k=1$, lo cual es consistente con los resultados del trabajo previo.

$$
\Delta \tau+k \sigma_{T}^{\max }=56,26 N_{R}^{-0,0412}
$$

Se observa en la Figura 5 cómo los datos experimentales colapsan en la misma línea tanto para $R=0$ como para $R=0,5$. Por tanto, el modelo basado en la tensión crítica de fatiga parece el camino correcto a seguir.

\section{CONCLUSIONES}

Este artículo ha estudiado el punto último de rotura en un material compuesto vidrio-epoxi con refuerzo de fibra unidireccional sometido a fatiga, teniendo en cuenta el efecto de la carga medio. Se han tenido en cuenta las componentes de tensión transversal y de cortadura en el plano como responsables últimas de la rotura por fatiga. Basándose en el modelo de fatiga y en el procedimiento analítico desarrollado, se pueden establecer las siguientes conclusiones:

- Los métodos de predicción convencionales curvas S-N -basadas en los esfuerzos de fatiga nominales a escala global- no son capaces de identificar los modos de fallo individual que existen en un material compuesto unidireccional sometido a cargas multiaxiales de fatiga. El modo de fallo por fatiga en un material compuesto con refuerzo de fibra unidireccional está gobernado por las tensiones locales y por el criterio de fallo asociado a estas tensiones. Por tanto, el método de predicción aquí propuesto se formula de acuerdo con las tensiones en cada lámina asociadas a los modos de fallo a escala local.

- Se comprueba que el método propuesto, que ya había sido capaz de predecir el comportamiento de un material compuesto con diferentes orientaciones de la fibra respecto a la dirección de la carga, es también capaz de evaluar la influencia que tiene la tensión media aplicada en la vida del material compuesto.

- Se concluye que, a medida que el ratio de esfuerzos aumenta, la vida de fatiga disminuye. Asimismo, para ángulos entre la fibra y la carga elevados, el modo de fallo predominante es el de rotura en la matriz por efecto de la carga transversal, siendo el comportamiento más estable.

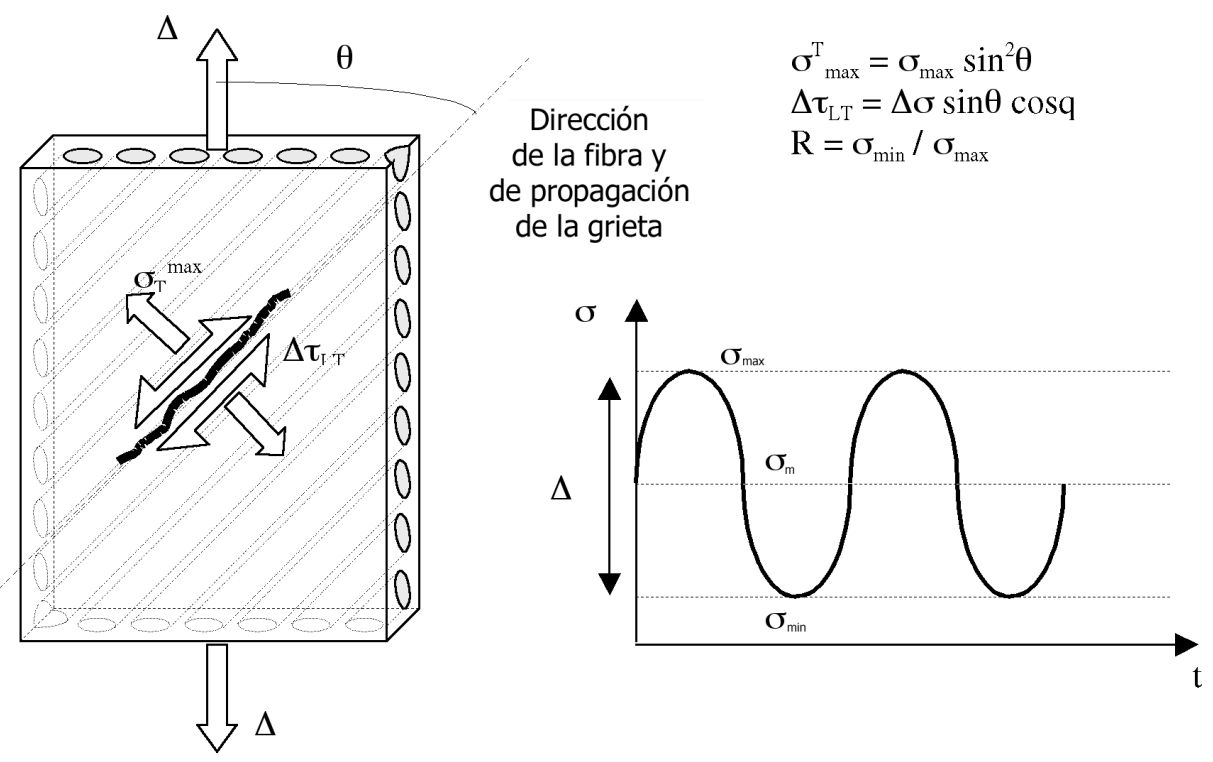

Figura 1. Parámetros de tensión cíclica en una lámina de material compuesto unidireccional sometida a fatiga. 


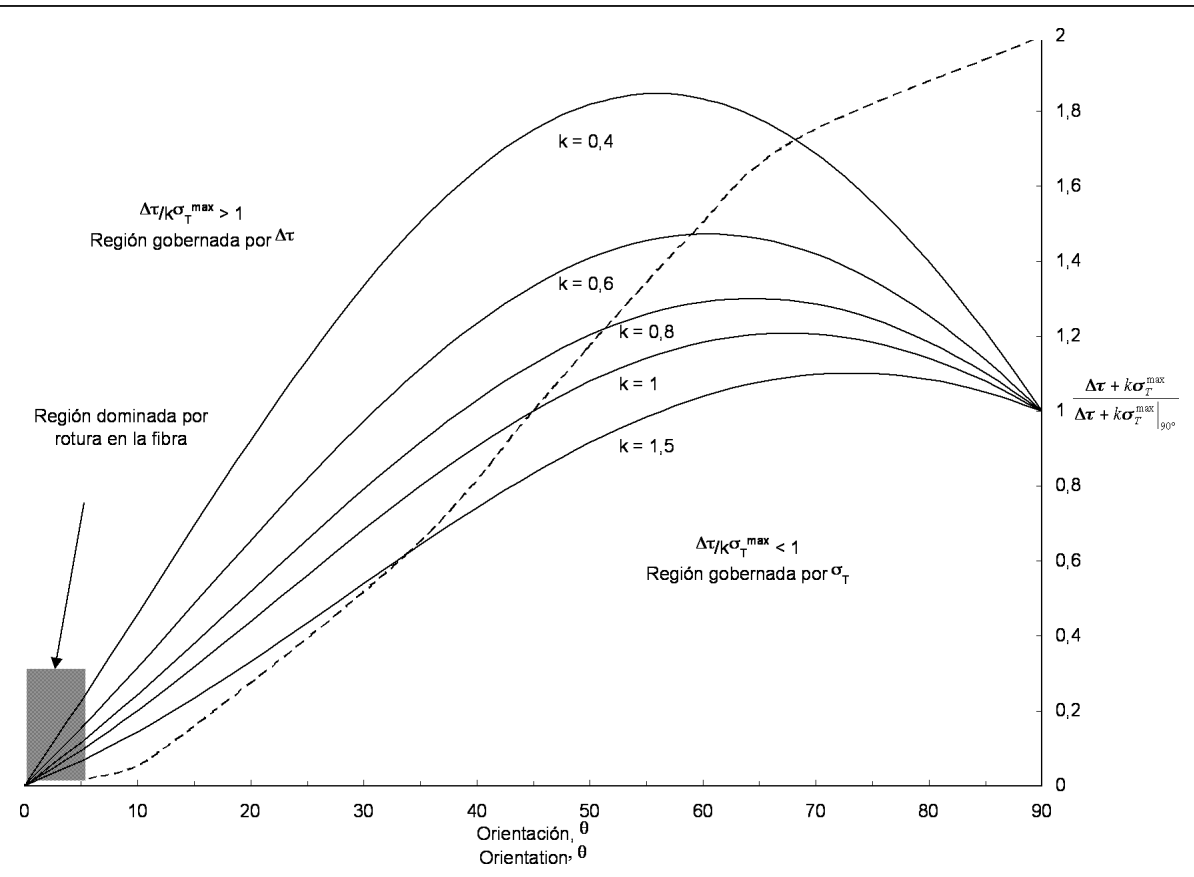

Figura 2. Variación de la función de tensiones de fatiga para un material compuesto unidireccional con respecto a la orientación de la fibra, $R=0$.

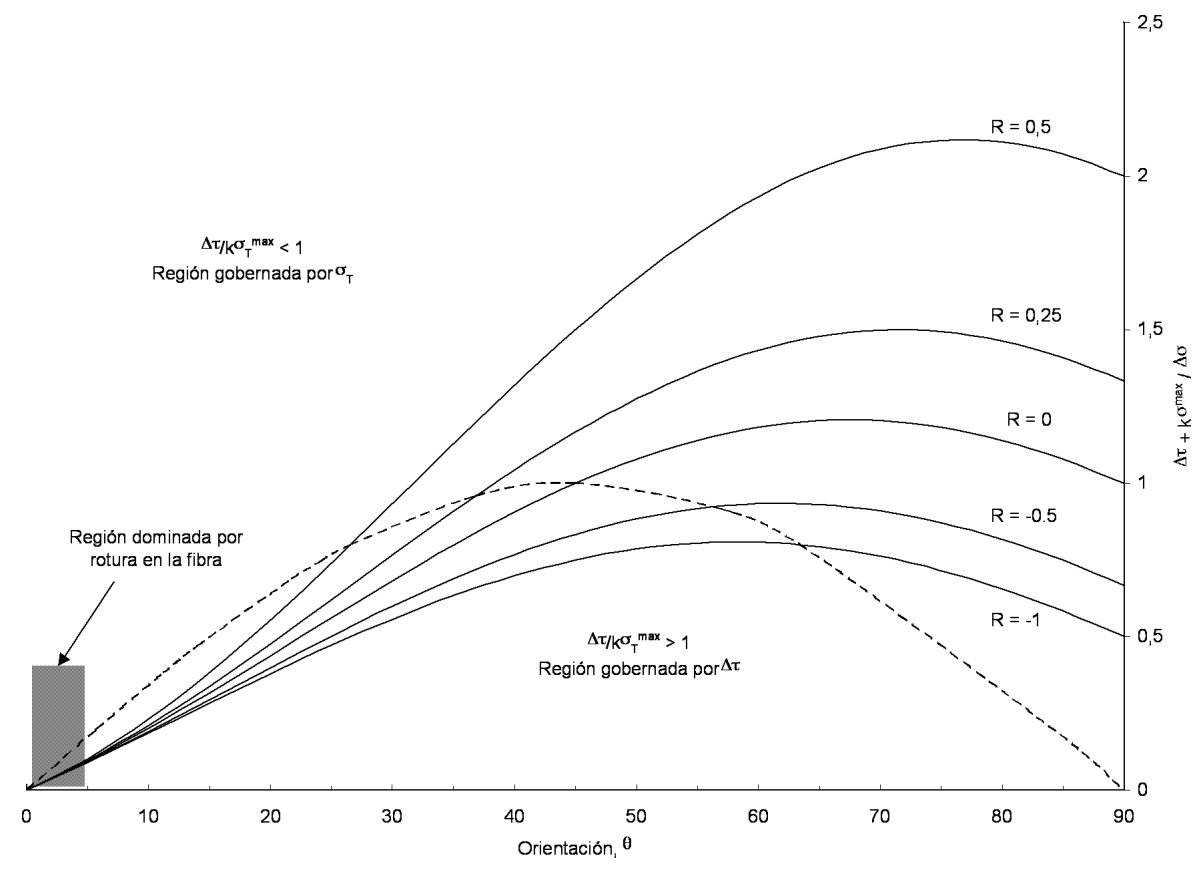

Figura 3. Efecto de la tensión media en la función crítica de tensiones de fatiga en un material compuesto unidireccional. 


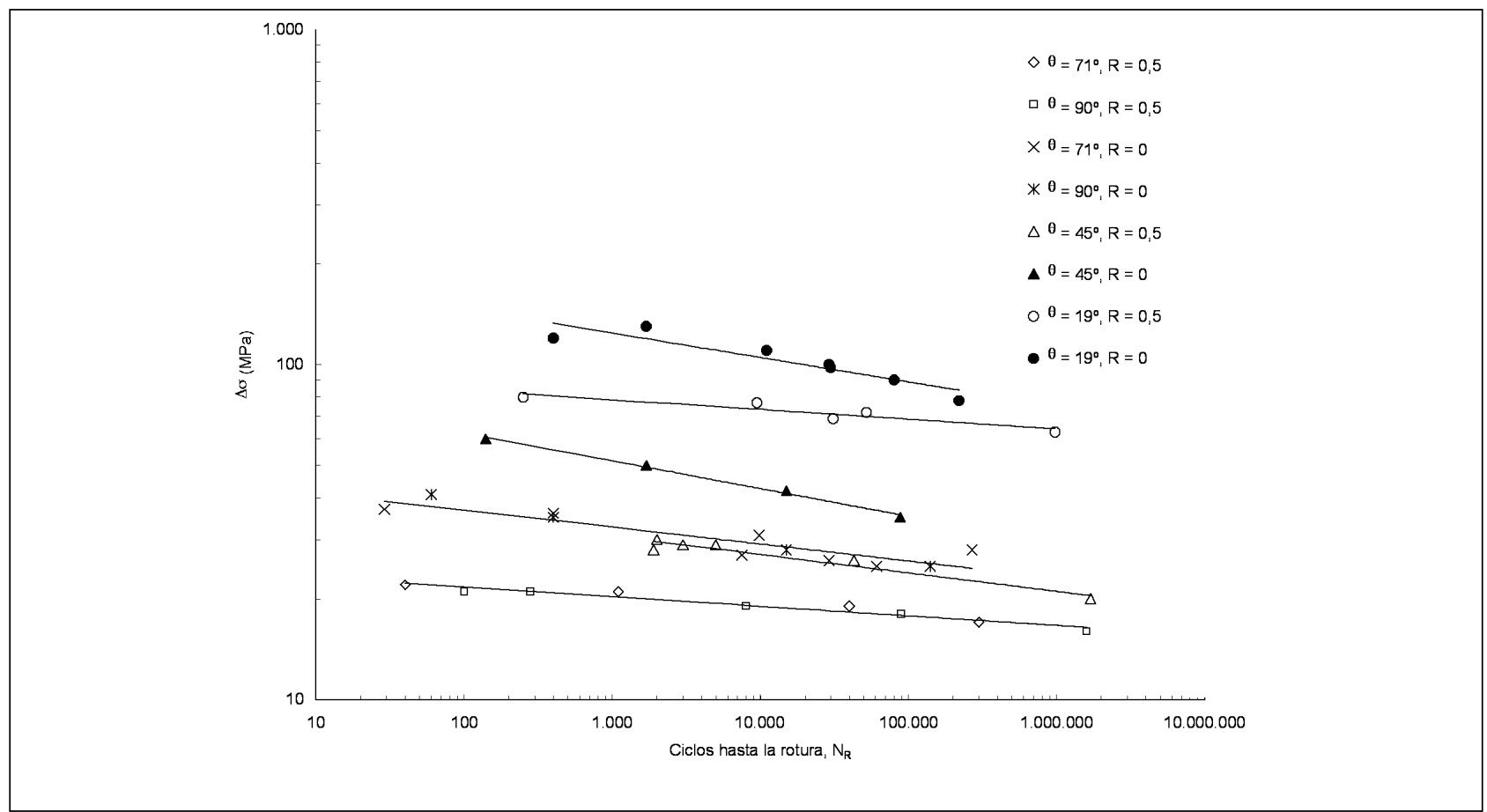

Figura 4. Influencia del ratio de esfuerzos $\mathrm{R}$ en la vida de fatiga de un material compuesto unidireccional vidrio-epoxi con distintas orientaciones (Fawaz y Ellyin, 1994).

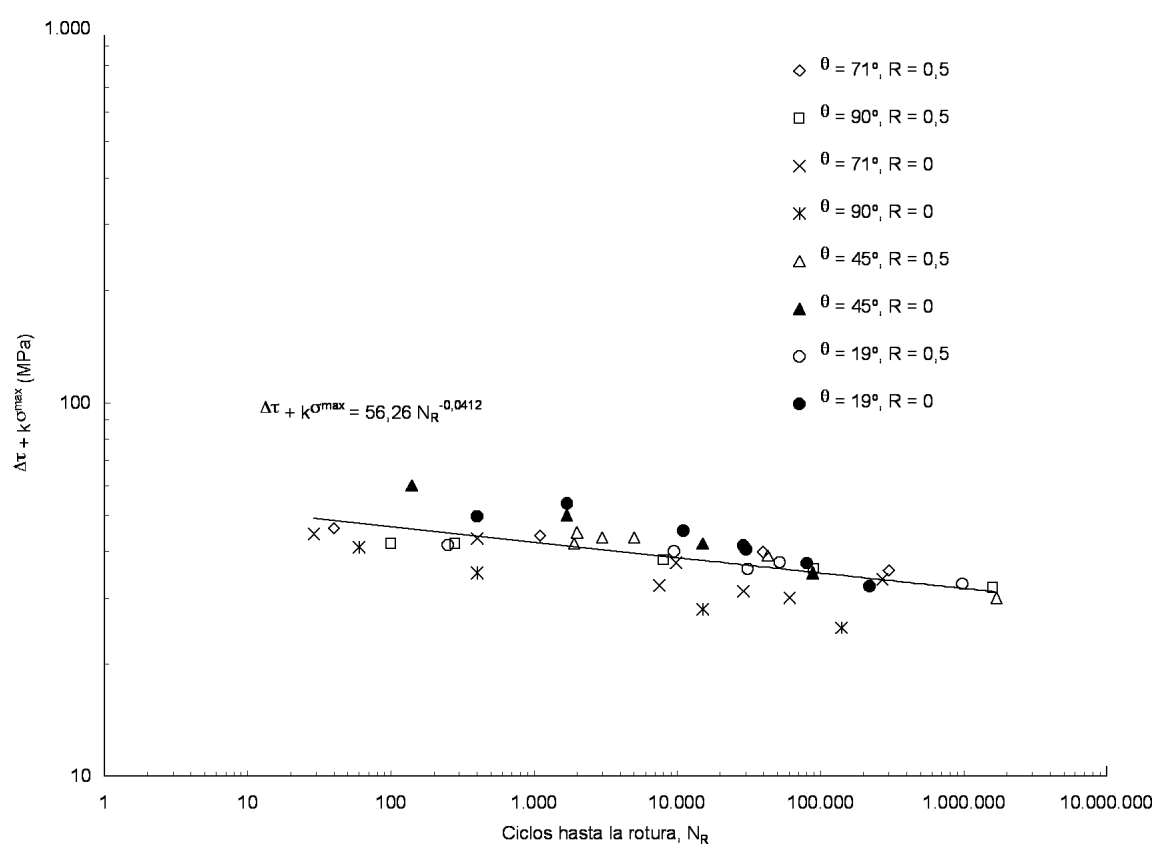

Figura 5. Función de tensión crítica de fatiga frente a la vida de fatiga en un material compuesto vidrio-epoxi unidireccional, para diversos ratios de esfuerzo $\mathrm{R}$ y diversas orientaciones. 


\section{BIBLIOGRAFÍA}

(1) Miner, M. A.: "Cumulative damage in fatigue", Journal of Applied Mechanics, vol. 12 (1945), p. 159.

(2) Hahn, H. T., Kim, R. Y.: "Fatigue bahavior of a composite laminate", Journal of Composite Materials, vol. 10 (1976).

(3) Revuelta, D.: "Predicción de la vida a fatiga en un material compuesto vidrio-epoxi unidireccional sometido a cargas cíclicas desviadas respecto de la dirección de la fibra", Mater. Contrucc., vol. 55, no 277 (2005), pp. 51-59.

(4) Fawaz, Z., Hellyin, F.: "Fatigue failure model for fiber reinforced materials under general loading condition", Journal of Composite Materials, vol. 28, no 4 (1994), pp. 1432-1451. 